\title{
Mise en évidence d'un cycle de croissance annuel chez Clarias gariepinus (Burchell 1822) (Pisces, Clariidae) du Bassin de la Luki (Zaïre)
}

\author{
S. Mutambue
}

J.N. Tourenq ${ }^{1}$

Mots clés : Poissons, Clarias, croissance, Zaïre

L'étude histologique des coupes des rayons osseux des nageoires pectorales de Clarias gariepinus pêchés entre décembre 1985 et février 1986 dans le bassin de la Luki (Zaïre) a montré que ces organes possèdent des marques cycliques pouvant être reconnues et envisagées comme critères d'âge.

Ces deux types de marques, probablement synchronisees avec l'alternance saison sèche/saison des pluies, sont bien individualisées : zone de croissance active (Z) et des lots de lignes d'arrêt de croissance (LAC).

Evidence for an annual growth cycle in Clarias gariepinus (Burchell 1822) (Pisces, Clariidae) from the Luki basin (Zaire) Keywords : Fish, Clarias, growth, Zaire.

A histological study was made of the bony rays of the pectoral fins of Clarias gariepinus, using fish caught between December 1985 and February 1986 in the Luki basin (Zaire). The study showed the presence of cyclic marks that could be used to age the fish. There were two distinct types of marks that alternated with the dry and rainy seasons, and could be used to distinguish a zone of active growth (2) and periods of arrested growth (LAC).

\section{Introduction}

L'étude de la dynamique des populations piscicoles indispensables à une bonne gestion du stock, ne peut se concevoir sans une bonne évaluation de l'âge des individus colonisant l'ècosystème étudié. Les travaux consacrés à la détermination d'âge, à la vitesse de croissance et à la longévité des Clariidae des eaux continentales africaines sont encore rares et très fragmentaires (El Bolock \& Koura 1959, El Bolock 1972, Micha 1973, Van der Wall 1975). Pour la détermination d'âge de Clarias gariepinus du bassin de la Luki, nous avons fait appel à l'étude histologique des premiers rayons épineux des nageoires pectorales qui, dans de nombreux cas, a permis la mise en évidence des marques des croissances

1. Laboratoire d'Hydrobiologie, URA 695 du C.N.R.S. Université Paul Sabatier, 118, Route de Narbonne, F-31062 Toulouse Cedex. cycliques, utilisables dans la détermination de l'âge individuel des Clariidae (Quick \& Burton 1984 ; Van der Waal 1975, Clay 1982).

\section{Matériel et méthodes}

Les poissons étudiés ont été capturés entre décembre 1985 et janvier-février 1986 dans le bassin de la Luki. Les techniques de pêche utilisées ont été décrites en détail dans Mutambue (1984 et 1985). La taille, la masse et le nombre de poissons analysés. sont donnés dans le tableau $\mathbf{I}$.

Plusieurs méthodes dont la lecture des opercules et des otolithes ont été utilisées pour essayer de mettre en évidence les différentes marques de croissance mais seule la lecture de coupes des rayons épineux de nageoires pectorales a donné des résultats satisfaisants. Ces rayons ont été prélevés sur chaque poisson et gardés à sec. Ils étaient ensuite nettoyés après avoir été trempés quelques minutes dans l'eau 
Tableau 1. Tailles (en $\mathrm{cm}$ ), poids (en g) et nombre de Clarias gariepinus étudiés.

\begin{tabular}{|c|c|c|c|c|c|c|c|c|c|c|c|}
\hline Tailles & 5 & 10 & 15 & 20 & 25 & 30 & 35 & 40 & 45 & 50 & \\
\hline Poids & & & & & & & & & & & Total \\
\hline$>10$ & 3 & 3 & 1 & & & & & & & & 7 \\
\hline 50 & & & 2 & & & & & & & & 2 \\
\hline 100 & & & & 5 & & & & & & & 5 \\
\hline 150 & & & & 4 & 3 & & & & & & 7 \\
\hline 250 & & & & & & 3 & & & & & 3 \\
\hline 300 & & & & 1 & 1 & 9 & & & & & 11 \\
\hline 20 & & & & & & 1 & & & . & & 1 \\
\hline 40 & & & & & & 5 & 1 & & & & 6 \\
\hline 40 & & + & & 2 & & . & 2 & & & & 4 \\
\hline 300 & & & & & & & 1 & 1 & & & 2 \\
\hline 60 & & & & & & & 1 & & & 3 & 4 \\
\hline 650 & & & & & & & 2 & 1 & & & 3 \\
\hline 700 & & & & & & & & 1 & & & 1 \\
\hline 750 & & & & & & & 2 & & & & 2 \\
\hline 800 & & & & & & & & 1 & & & 1 \\
\hline 900 & & & & & & & & 2 & & & 2 \\
\hline 950 & & & & & & & & 3 & & & 3 \\
\hline 1100 & & & & & & & & 3 & & & 3 \\
\hline 12100 & & & & & & & & & 3 & & 5 \\
\hline Total & 3 & 3 & 3 & 12 & 4 & 18 & 9 & 12 & 5 & 3 & 72 \\
\hline
\end{tabular}

bouillante et décalcifiés par bains successifs dans des solutions d'acide nitrique ( 36 heures à $5 \%$, puis 24 heures à $3 \%$ pour les grandes pièces; 24 heures dans des solutions à $3 \%$ pour les pièces de petites tailles).

Après un rinçage à l'eau courante durant 24 heures, certaines de ces pièces étaient débitées en coupes fines de $16 \mu \mathrm{m}$ d'épaisseur au moyen d'un microtome à congélation. D'aut res échantillons de $5 \mathrm{~mm}$ d'épaisseur étaient prélevées au moyen de bistouri.

Ces différentes coupes étaient pratiquées à la base du rayon osseux comme l'ont préconisé Marzolf (1955), Davis (1977), Meunier et al. (1979) (fig. 1, $2,3: 1$ ).

Les coupes fines ont été colorées à l'hématoxyline d'Ehrlich durant 20 minutes (Klevezal \& Kleinnenberg 1967, Smirina 1974, Meunier et al. 1979) et montées entre lames et lamelles dans une résine aqueuse avant leur observation au microscope photonique.

Les échantillons de $5 \mathrm{~mm}$ étaient déshydratés dans l'éthanol, puis ont reçu une mince couche d'or palladium (30 minutes environ) à leur surface. Ils étaient ensuite examinés au moyen du microscope à balayage JEOL JS MT 200 .

\section{Résultats}

Les différentes sections des rayons observés sont grossièrement triangulaires et présentent cinq régions bien distinctes : une antérieure ou apex (AP), deux latérales postérieures ou ailes $(A L)$, une centrale (CM) et une médiopostérieure située entre les deux ailes (fig. 2 et $3: 1$ ).

Ces rayons sont pourvus de cavités médullaires plus ou moins développées. Les contours de ces dernières sont souvent remaniés et constitués d'ostéones (Meunier et al., 1979). Le tissu osseux, de nature compacte, montre des ostéocytes et est traversé par des canaux vasculaires radiaires ainsi que par des canaux longitudinaux (CL).

L'analyse histologique de ces coupes a permis de montrer que ces rayons possèdent des structures cycliques se manifestant par l'alternance des zones $(Z)$ et des lignes hématoxylinophiles (LH). Ces zones (Z) correspondent à une période d'ostéogénèse active et les lignes hématoxylinophiles ( $\mathrm{LH}$ ) à un ralentissement de la croissance sinon à un arrêt de l'ostéo- 


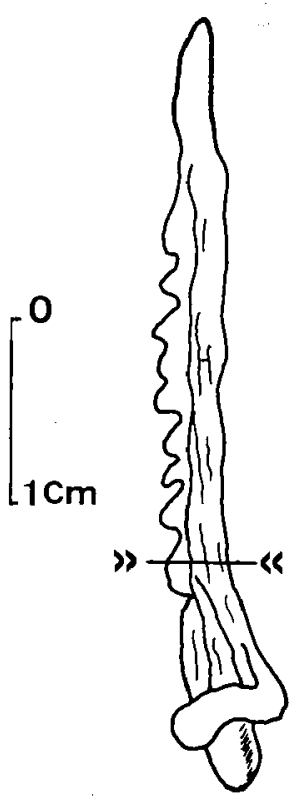

Fig. 1. Epine de la nageoire pectorale de Clarias gariepinus.

génèse (Menon 1950, Bagenal 1974, Daget \& Leguen 1975, Castenet et al. 1977, Francillon 1979, Meunier et al. 1979, Meunier et Pascal 1980, Meunier 1983, Castanet 1985, Lecomte et al. 1985, 1986) (fig. 3).

Excepté dans la région médiopostérieure, ces lignes d'arrêt de croissance (LAC) sont séparées par un intervalle assez large. Ce dernier se réduit au fur et à mesure que les animaux vieillissent. Certaines de ces lignes sont discontinues. Ces fausses LAC sont les « false rings » des auteurs anglo-saxons dont l'origine la plus fréquente serait due à un « stress » subi par le poisson (variation brutale de l'environnement physico-chimique, maladies...) (Philippart 1971, Gheno 1975).

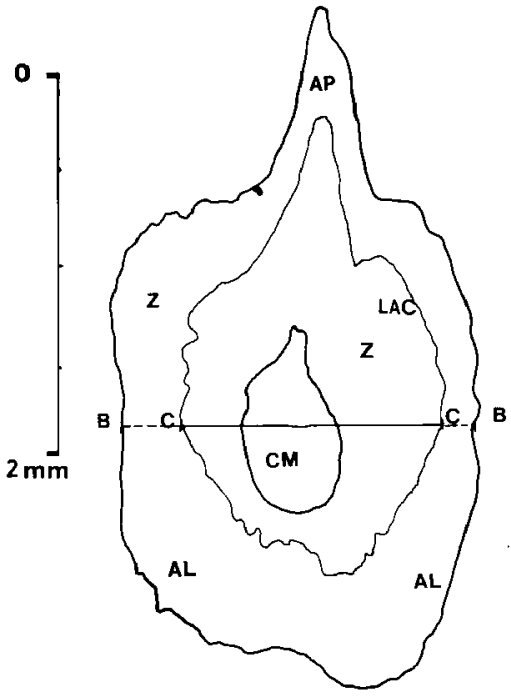

Fig. 2. Schéma d'une coupe d'épine de nageoire pectorale. $A P=$ Apex. $\mathbf{A L}=$ Ailes. $\mathbf{C M}=$ Canal médullaire. $\mathrm{LAC}=$ Ligne d'arrêt de croissance. $B B=$ Largeur totale de l'épine. $\mathrm{CC}=$ Largeur du permier annulus.

Chaque LAC se compose d'un lot de deux à six lignes hématoxylinophiles relativement serrées et parallèles (photos 2 et 3 ). Ce phénomène est probablement lié à des reprises temporaires de la croissance durant la période de ralentissement ou d'arrêt de croissance à la faveur de conditions climatiques clémentes.

\section{Discussion}

Il est difficile actuellement de donner une explication exacte des relations existant entre la structure histologique observée et le cycle de croissance de Clarias gariepinus. L'étude expérimentale de la croissance de ces animaux maintenus en conditions 

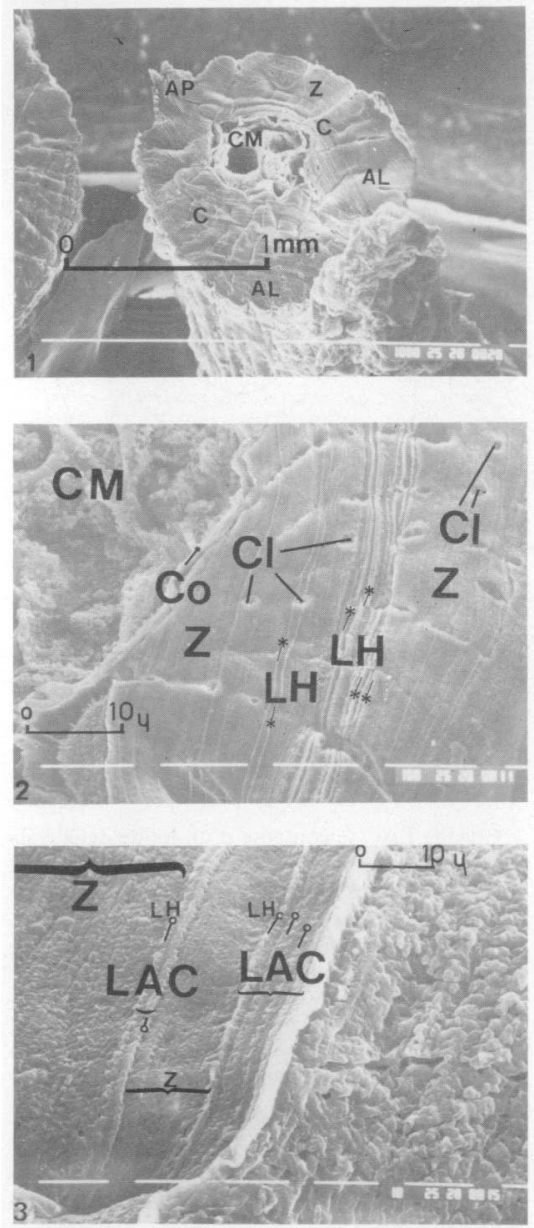

naturelles et artificielles (élevage contrôlé) serait nécessaire pour préciser cette question. Toutefois, la comparaison de nos données (à paraître) à celles obtenues par El Bolock \& Koura (1959) dans le bassin d'élevage au Caire (Egypte), El Bolock (1972) dans un lac égyptien, Micha (1973) dans les étangs de barrage et de piscicultures, Pivnicka (1974) dans le lac Kariba (Zambie) et Van der Wall (1975) dans les rivières Elands et Olifands (Prétoria, S.A.) laisse penser que les lignes d'arrêt de croissance observées correspondent à des lignes d'arrêt de croissance annuelle. Les études en cours doivent préciser la période de leur formation.

\section{Conclusion}

A partir d'une technique de préparation simple à réaliser le résultat essentiel de la série d'observation décrites dans ce travail est la mise en évidence de l'alternance de zone de croissance active pouvant être liée à l'activité métabolique générale intense de la belle saison (Castanet et al. 1977, Meunier 1983, Castanet 1985, Lecomte et al. 1985) et de groupe de LAC marquant le ralentissement sinon l'arrêt de la croissance en liaison avec la réduction du métabolisme (Lecomte et al. 1986).

Daget \& Leguen (1975), Castanet et al. (1977), Bourlière (1980), Castanet (1985), Lecomte et al. $(1985,1986)$ ont montré que cette alternance est calquée sur celle de la saison des pluies/saison sèche.

Nous pouvons supposer que la mise en place des LAC a lieu durant la grande saison sèche (mi-mai à mi-septembre).

Daget (1956) et Garrod (1959) lient la présence d'annuli (LAC) aux périodes d'activité sexuelle.

Comme Hopson \& Hopson 1965), Le Roux (1961), Durand \& Loubens (1969), nous pensons que la reprise de la croissance accélérée (zone $\mathrm{Z}$ ) est

Fig. 3. - 1. Coupe d'épine de nageoire pectorale de Clarias gariepinus. Légende voir fig. 2.

- 2. Détail de la coupe transversale du rayon épineux de la nageoire pectorale d'un Clarias gariepinus observé au microscope électronique à balayage. $\mathrm{CM}=$ Canal médullaire. $\mathrm{Co}=$ Contour remanié du canal médullaire. $\mathrm{CL}$ = Canaux longitudinaux. $Z=$ Zone de croissance active. $\mathbf{L H}=$ Lignes d'arrêt de croissance : lignes hématoxylinophiles. $\mathbf{L A C}=$ Zone d'arrêt de croissance.

- 3. Détail de la structure de la zone d'arrêt de croissance LAC (lots de lignes hématoxylinophiles LH). 
la reprise de la croissance accélérée (Zone Z) est provoquée par l'augmentation des ressources nutritives du milieu.

Une dérive plus importante provoquée par les forts débits pendant la saison des pluies peut être une hypothèse logiquement envisageable. Cette hypothèse vérifiée permettrait l'élaboration d'un modèle de croissance pour cette espèce économiquement intéressante.

\section{Travaux cités}

Bagenal (T.B.). 1974. - The ageing of fish. Proc. Intern. Symp. Univ. of Reading, 19-20 June 1973. Unwin Brother's Ltd. : $234 \mathrm{p}$.

Boet (P.). 1981. - Eléments d'écologie du poisson-chat : Ictalarus melas (Rafinesque, 1820), du Lac de Créteil ; structure et dynamique de la population, exploitation des ressources alimentaires et production. Thèse de doctorat $3^{e}$ cycle. Univ. Pierre et Marie Curie, Paris 6: $123 \mathrm{p}$.

Boulière (J.). 1980. - La périodicité de la croissance dans le monde animal et son intérêt écologique. Bull. Soc. Zool. Fr., $105: 273-276$.

Castanet (J.). 1985. - La squelettochronologie chez les reptiles. I. Études expérimentales de la croissance chez les lézards et les tortues. Ann. Sci. Nat. Zool., $13^{\circ}$ ser., $7: 23-40$.

Clay (D.). 1982. - A comparison of different methods of age determination in the Sharptooth cat fish Clarias gariepinus. J. Limnol. Soc. Sth. Afri. 8 (2) : 453-482.

Castanet (J.), Meunier (F.J.) \& De Ricqles (A.). 1977. - L'enregistrement de la croissance cyclique chez les vertèbrés poikilothermes : données comparatives et essai de synthèse. Bull. Biol. Fr. Belg., 111 : 183-202.

Daget (J.). 1956. - Mémoire sur la biologie des poissons du Niger moyen .II. Recherches sur Tilapia zilli. Bull. I.F.A.N., $18 \mathrm{~A}, 1: 165-223$.

Daget (J.) \& Leguen (J.C.). 1975. - Les critères d'âge chez les poissons. In M. Lamotte et F. Bourlière : 253-289. Problème d'écologie : la démographie des populations de vertébrés. Masson, éd. 443 p.

Davis (T.L.O.) . 1977, - Age determination and growth of the freshwater catfish Tandannus iandannus. Austr. Jour. of Mar. \& Freshwater Res., 28 (2) : 119-171.

Durand (J.R.) \& Loubens (G.). 1969. - Croissance en longueur d'Alestes baremoze (Joannis, 1835) (Poissons Characidae dans le Bas Chari et le lac Tchad. Cah. O.R.S.T.O.M., ser. Hydrobiol., vol. III (1) : 60-105.

El Bolock (A.R.). 1972. - The use vertebrae for determining age and growth of the Nile catfish Clarias lazera (Cuvier et Valencienne) in the Are. Bull. Inst. Oceamogr. Fish. Cario, $\mathrm{N}^{\circ} 2: 53-82$.

El Bolock (A.R.) \& Koura (R.). 1959. - Observation on age, growth and feeding habits of Clarias lazera (Cuvier et Valencienne) in barrage experimental ponds. Note Mem. Hydrogiol. Dep. Cario, $\mathrm{N}^{\circ} 56: 1-17$.
Francillon (H.). 1979. - Etude expérimentale des marques de croissance sur les humérus et les fémurs de tritons crêtés ( $T r i$. turus cristatus cristatus Laurenti) en relation avec la determination de l'âge individuel. Acta Zool., $60: 223-232$.

Hopson (A.J.) \& Hopson (J.). 1965. - Winter scale rjngs in Lates niloticus (Pisces, Centropomidae) from Lake Chad. Nature: 1013-1014.

Garrod (D.G.). 1959. - The growth of Tilapia esculentia in Lake Victoria. Hydrobiol. 12 (4) : 268-298.

Gheno (Y.). 1975. - Nouvelle étude sur la détermination de l'âge et de la croissance de Sardinello aurita Val dans la région de Pointe Noire. Cah. O.R.S.T.O.M. sér. Océanogr., vol. XIII. (3) : 251-262.

Klevezal (G.A.) \& Kleinnenberg (S.E.). 1967. - Détermination de l'âge des mammifères d'après les structures tamellaires des dents et des os. Trad. du Russe (1969), Israel Prog. Scient. Transl. Jerusalem : $142 \mathrm{p}$

Lecomte (F.), Meunier (F.J.) \& Rojas-Beltran (R.). 1985. Mise en évidence d'un double cycle de croissance annuel chez un Silure de Guyane, Arius couma (Val. 1839) (Teleostei, Siluriformes, Aridae) à partir de l'étude squelettochronologique des épines des nageoires. C.R. Acad'. Sci. 300: 181-184.

Lecomte (F.), Meunier (F.J.) \& Rojas-Beltran (R.). 1986. Données préliminaires sur la croissance de deux Téléostéens de Guyane : Arius proops (Ariidae, Siluformes) et Leporinus friderici (Anostonidae, Characoidei). Cybium, 10 (2) : $121-134$.

Le Roux (J.). 1961. - Growth of Tilapia mossambica in some Transvaal impondments. Hydrobiol., 18, 1-2: 165-175.

Marzolf (R.C.). 1955. - Use of pectoral spines and vertebras for determining age and rate of growth of the Channel catfish. $J$. of wildlife manag., 10 (2) : 243-249.

Menon (M.D.). 1950. - The use of bones, other than otoliths in determining the age and growth rate of fishes. $J$. Cons., 16 (3) : $311-340$.

Meunjer (F.J.). 1983. - Les tissus osseux des Osteichthyens. Structure, genèse, croissance et évolution. Archives et documents, micro-édition, Inst. Ethnol., SN 82-600-328:200 p.

Meunier (F.J.), Pascal (M.) \& Loubens (G.). 1979. Comparaison de méthodes squelettochronologiques et considérations fonctionnelles sur le tissu osseux acellulaire d'un Ostéichthyen du Lagon néocalédonien. Aquaculiure, 17 : 137-157,

Meunier (F.J.) \& Pascal (M.). 1980. - Quelques données comparatives sur la croissance périodique des tissus squelettiques chez les Ostéichthyens. Bull. Soc. 20ol. Fr., 105 : 337-342.

Micha (J.C.). 1973. - Etude des populations piscicoles de l"Ubangui et tentatives de sélection et d'adaptation de quelques espèces à l'étang de pisciculture. C.T.F.T., Nogent-surMarne : $110 \mathrm{p}$.

Mutambue (S.). 1984. - Contribution à l'étude de l'écologie de la rivière Luki (sous-affluent du fleuve Zaïre) : Bassin versant - Poissons). Thèse doctorat $3^{e}$ cycle, Univ. Paul Sabatier, Toulouse : $214 \mathrm{p}$. 
Mutambue (S.). 1985. - Premières données sur le peuplement des poissons de la rivière Luki. Cybium, 9 (11): 17-28.

Philippart (J.Cl.). 1971. - Age et croissance de la vandoise, Leuciscus leuciscus (L) dans l'Ourthe et la Berwine. Ann. Soc. Roy, Zool. Belg., 101 (1-2): 77-140.

Pivnicka (K.). 1974. - La croissance des poissons d'eau douce africains : Revue bibliographique et essai de généralisation. In B. de Mérona, J.M. Ecoutin, 1979 : Réunion de travail sur la limnologie africaine. Nairobi : 139 p.
Quick (A.J.R.) \& Burton (M.N.). 1984. - Age and growth of Clarias gariepinus (Pisces, Clariidae) in the P.K. le Roux Dam South Africa. S. Afr. J. Zool., $19: 37-45$.

Scheffer (V.B.). 1950. - Growth layers on the teeth of Pinnidedia as indicator of age. Science, $112: 309-311$.

Smirina (E.M.). 1974. - Annual layers in bones of Rana temporis. Zool. Zh. 51 : 1529-1534.

Van der Waal (B.C.W.). 1975. - Age and growth studies of Clarias gariepinus in the Transvaal. S.A.J. Fish. Biol., 7 : 227-233. 